\title{
Aether, fields \& energy dynamics in living bodies - Part I
}

\author{
K. E. Thorp ${ }^{1}$, James A. Thorp ${ }^{2 \star}$, Paul R. Walker ${ }^{3}$
}

'Department of Radiology, Sparrow Health System, Lansing, MI 2Department of Obstetrics and Gynecology, Division of Maternal Fetal Medicine, Sisters of St. Mary's Health System, St. Louis, MO.

${ }^{3}$ BSME, MSEE

\section{Introduction}

At the turn of the $20^{\text {th }}$ century medicine and physics seemed to be heading in two opposing if not mutually exclusive directions: medical science had asserted the primacy of the cell and had set out to integrate known chemical principles into cellular functioning; physics, on the other hand, had broken through the barriers of classical Newtonian mechanical laws and established the energetic basis of all physical substance. Something was bound to give.

Medical scientists brimmed with confidence. Some, like English physiologist Ernest Starling, enthusiastic proponent of the new science, claimed that medicine would gain complete control over the human body; others predicted that diseases like infection would be eradicated. A century later western societies face an epidemic of chronic diseases unlike ever before described in human history. Medical science has perfected the art of managing disease but there is not the least indication that it is any closer to discovering a cure for any of the chronic diseases than a century ago.

And to compound matters, for decades modern physics has been mired in an intractable controversy regarding the source and nature of the energetic phenomena detected throughout the cosmos. Visible matter is now known to comprise less than five percent of the universe with the rest being occupied by what is called dark energy and dark matter. Modern physics is unable to answer even the most basic questions involving either the origins or nature of such phenomena. Nor have physicists been able to bridge the century-old conceptual gap between the two reigning explanatory frameworks, relativity and quantum mechanics.

Over two millennia ago Greek philosopher Aristotle argued for the impossibility of empty space and advanced the notion of an all-encompassing medium he called aether, an invisible and insensible substance which he imputed to be the source of all order and motion in the celestial realm. Physicists from Newton through Maxwell and Thomson used the aether concept to explain not only how forces like gravity asserted their effects but how light and electromagnetic waves propagated through space. Aether was rejected by physicists at the turn of the $20^{\text {th }}$ century without ever refuting its existence.

By the late decades of the $20^{\text {th }}$ century the cellular paradigm had become so impossibly complex that few could say with any confidence they understood what actually happened inside the body. Since the 1950 senetic mechanisms have become the default explanatory framework despite the fact they fail to account for a wide range of biological phenomena. As early as the 1970s scientists like Nobel laureate Macfarlane Burnet, in Genes, Dreams and Realities, sounded the alarm on the failing paradigm arguing that cellular mechanisms were only descriptive and had little explanatory relevance in terms of health and disease.

For most of the $20^{\text {th }}$ century the heart was conceived as a mechanical pump that propelled blood forward through the arteries during its systolic contraction phase. In the 1980 s the pump model was overturned when negative 
pressure, i.e., a vacuum, was discovered in the ventricular cavity early in the diastolic filling phase. Researchers soon realized a suctional force must be responsible for the forward flow of blood into the ventricles. Spiral flow currents were subsequently found in large and mediumsized arteries which can only be explained on the basis of a suctional force within the arteries. In parallel with these developments heart rate variability patterns were found to be a leading predictor of health and all-cause mortality with diminished outward movement of the heart wall, i.e., diastolic dysfunction, a prominent feature in many chronic diseases. What does all this indicate?

It has been nearly three decades since most of these discoveries were made and yet, to date, the heart is still widely conceived as a mechanical pump. There has been no synthesis of these emergent facts into a new model of cardiac function. The identification of a vacuum in early diastole can only indicate the presence of an active force within the ventricular cavity. Along with other evidence this suggests that the heart, aside from moving blood through the vascular system, is responsible for generation of a body-wide energy field and, moreover, that the outward movement of the heart wall during diastole can be regarded as proxy to the influx of energy substance into the cardiac chamber.

Other peculiarities of cardiac anatomy and function come to bear. What purpose would large iron stores in heart muscle and blood serve if not as the substrate for a magnetic field? For over a century it has been claimed that electrical currents flowing through nerves over the surface of the heart induce its contraction despite the fact that the heart possesses intrinsic rhythmicity and continues to beat in the absence of nerves. Evidence points in a different direction.

It has been known for more than a century that exposure of iron to an external electric field induces formation of a magnetic field. Faraday's Law suggests that the spiral flow of electrical currents in the blood should induce a magnetic field. How else can one explain electromagnetic currents, for example, detected by magnetoencephalography in the cerebrospinal fluid? Evidence from decades of magnetic resonance imaging suggests the whole body is an organized magnetic field.

In recent decades an increasing number of physicists have argued against the notion of empty space and, instead, for the necessity of some kind of universal medium, an aether-like substance, to account not only for phenomena like dark matter and dark energy but the very existence of physical substance. Aether has become an inescapable reality in the dynamics of the cosmos.

In this three part paper we review the development and evolution of the aether concept and, for the first time, show its pivotal role in the generation and propagation of a complex body-wide field consisting of three intertwined and interconvertible primary energy forms: magnetic, taking origin in the vascular system; luminous, deriving from external sunlight, generated in the interstitial fluid compartment beneath the skin, and orchestrated by the kidneys; and the dielectric, originating at the cellular and molecular level and mediated primarily by electro-ionic mechanisms. We provide numerous examples of bodily phenomena in both health and disease that can only be explained on the basis of this organized aether field. In this first part we examine the history and evolution of the aether concept.

\section{Protean Aether}

The first systematic arguments in support of aether are presented by Aristotle in his work On the Heavens from about $350 \mathrm{BC}[1,2]$. His writings on the necessity of aether were, at least in part, directed toward the doctrine of atomism which had been advanced a half-century earlier by Democritus and Leucippus of the School of Abdera in Thrace. Atomists argued that the natural world was composed of two primary and opposing conditions: invisible and indestructible atoms, which freely move about and combine into the manifold visible forms of nature, and a hypothetical contrary state of nothingness known as the void or vacuum.

Western intellectual history constitutes a back-and-forth sally between two opposing schools of thought, atomism and continuum theory, the latter of which Aristotle is a leading proponent. This assumes significance in that the birth of the science movement in the $17^{\text {th }}$ century coincides with systematic repudiation of Aristotle's philosophy and rise of modern atomistic philosophy as propounded in the writings of Descartes. Thus, issues surrounding the existence (or non-existence) of aether transcend the physical sciences and involve metaphysics, that branch of philosophy concerned with first principles.

In On the Heavens Aristotle advances arguments in favor of aether and concludes that physical substance alone is insufficient to explain the cosmos: 'there is something besides the bodies nearby and around us ... having a more honorable nature to the degree that it is distinct from the world at hand.' Here we must anticipate what Aristotle is attempting to explain with his aether concept. It is not just a substance which fills the emptiness of space but, equally, possesses causal priority and engenders a multitude of secondary phenomena. We should not conflate any of his comments on the 'heavens' as pertaining strictly to spatial composition. When Aristotle speaks of aether as the 'first body' or 'divine body' he literally means that it gives rise to a plethora of downstream effects.

His two most compelling arguments involve repudiation of the void and the necessity of aether to explain the circular motions of celestial bodies in the night-time sky. 
Aristotle acknowledges he is constrained by knowledge of the day and his adjudications are based largely on belief. We briefly consider his arguments.

Aristotle's refutation of the void has to do with the impossibility of what is called action-at-a-distance. Wherever cause and effect occur between two entities they must always interact within a commonly shared medium. On this basis he argues that empty space cannot exist. Any apparently empty region must, instead, be filled with a subtle, transparent medium, the aether, which can be neither sensed nor grasped. The fundamental premise of continuum theory is the seamless continuity of the cosmos.

While the second argument Aristotle advanced in favor of aether, the circular nature of celestial motions, appears to be based on false premises, his conclusions are intuitively sound. Aristotle incorrectly assumed a geocentric perspective, i.e., that celestial bodies circled the earth, and that their motions are circular when in fact they are ellipsoid. Nonetheless the basic principles he distilled remain valid in light of current knowledge.

Aristotle used the aether concept not to explain celestial bodies per se but, rather, celestial motion, i.e., as the prime mover and source of unceasing heavenly movement. Circular motion has several features that bolster his conclusions. First it represents a perfect balance of forces, the centrifugal force that tends to push objects away from the center, and the centripetal which draws an object toward the center. The second property of circular celestial motion which might lead one to draw such conclusions is that it recurs at regular intervals and possesses predictable periodicity.

Aristotle's designation of circular celestial motion as 'eternal' simply meant that the same pattern recurs again and again. Since the motion persists without alteration it could be also described as immutable or invariant. And given that it seems to be characteristic of all heavenly bodies one might regard it as a universal attribute and representing a principle upon which the organization of the cosmos is based. The fact that Copernicus later deduced that planets orbit around the sun or Kepler showed the elliptical nature of planetary motion does not invalidate Aristotle's conclusions concerning the existence of aether.

Even though Copernicus' recognition of the heliocentric nature of planetary motion did not nullify Aristotle's aether claims, it nonetheless had significant consequences. In describing aether Aristotle limited its actions to the celestial sphere 'out there' and not 'here' within the sublunary or earthly region. But the fact that planets like earth orbit the sun in recurrent and predictable patterns would seem to imply that aether must assert its effects locally upon planetary bodies. This was not lost on $17^{\text {th }}$ century scientists. It became increasingly apparent that beyond explaining heavenly motions aether was needed to explain terrestrial phenomena as well.

Following Aristotle's lead Newton dismissed action-ata-distance, calling it 'inconceivable' and 'so great an absurdity that I believe no man who has in philosophical matters a competent faculty of thinking can fall into it' [3]. Analogous to how Aristotle used aether to explain the motion of celestial bodies, Newton regarded it as indispensible to explain long-distance effects of the gravitational force and a necessary means by which the sun held planetary bodies in their orbits. Equally, aether was essential to explain light and its various attributes such as refraction $[4,5]$. Based on the centrality of aether Newton coined the terms absolute space and absolute time to indicate their existence independent of all other external cosmic objects [6].

Experiments were performed to investigate the nature of the invisible medium. In 1660 Robert Boyle, using a vacuum pump, evacuated air from a glass container and demonstrated that the ringing of a bell inside the jar could no longer be heard [7]. The transmission of sound required the medium of air. And yet the 'empty' space still transmitted light. Much of the opinion concerning the insensible aethereal substance was drawn through analogical reasoning: rays of light are to aether what sound is to air, or ocean waves to water. Based on such logic the necessity of a conductive medium seemed selfevident.

Early on the notion of wave-like oscillation or vibratory motion was incorporated into the scientific debate. The transmission of sound is affected in wave-like manner by alternate compression and rarefaction of the air medium. It isn't sound per se that travels through the air but, rather, the oscillatory activity. In this sense, sound is an epiphenomenon created by perturbation of the air medium, a momentary disturbance that spreads through space transported by an undulating medium.

Just as sound is carried through air, and ocean waves on water, perhaps, it was further reasoned, light might be carried in the same manner by the aether and, moreover, light, like sound, might be secondary to vibrations of the aethereal medium. Just as the pitch of sound increases in proportion to its frequency of propagation through the medium, it was reasoned that light must represent miniscule vibrations that propagate through the aether at exceedingly high speed. This notion was first proposed by Robert Hooke in the 1660s. In following decades Christiaan Huygens advanced the notion of the 'luminiferous' or light-bearing aether as being essential for the propagation of light [8].

But the use of analogy by $17^{\text {th }}$ century scientists, based on likening an unknown to a known entity, had limitations. While Newton theorized that light was composed of 
invisible atomic particles or 'corpuscles,' scientists like Huygens and Thomas Young argued in favor of their wavelike nature. In either case it was theorized that the particles or waves displaced the aether medium through which it traveled (not unlike sound waves) and aether was thus conceived as possessing density, elasticity and masslike attributes, i.e., an 'elastic solid,' that would offer resistance and tension not unlike air with respect to sound waves. This assumption would eventually be the undoing of luminiferous aether at the end of the $19^{\text {th }}$ century.

\section{Light, Electricity \& Magnetism}

By the turn of the $19^{\text {th }}$ century Newton's corpuscular theory of light was on the ropes. The final blow came when Thomas Young pointed out optical experiments whose results could only be explained by wave theory. And wave theory explained phenomena like reflection and refraction better than atomic theory [9]. Young embraced the aether concept and argued that it was 'undeniably proved' by the newly discovered phenomenon of electricity. He was among the first to draw attention to similarities between electricity and light: 'the rapid transmission of the electrical shock shows that the electric medium is possessed of an elasticity as great as is necessary to be supposed for the propagation of light.' The next conceptual breakthroughs involved studies on the nature of electromagnetism.

In the 1820s Michael Faraday set about to study the induction of electric currents and in the process made linchpin discoveries regarding relationships between electricity and magnetism that would eventually pave the way for a new conception of light [10]. In one experiment he wound two different coils of wire around a torusshaped iron ring with the wire on the right side connected to a voltage meter and the wire on the left side connected to a battery through a switching device. The meter registered momentary deflections whenever the switch was opened or closed indicating current induction. When the iron torus was removed and the wire coils were separated by 'empty' space an induction effect was still produced when the switch was opened or closed. And when the battery on the left side was removed altogether, Faraday found that simply sweeping a magnet in and around the wire coil induced current flow and deflection of the voltage meter.

To explain such phenomena Faraday introduced the concept of the field, a three-dimensional causal nexus surrounding the flowing electric currents. If iron filings are strewn upon a sheet of paper and a magnet is brought underneath, they spontaneously rearrange themselves into curvilinear lines of magnetic force whose direction at any point coincides with the intensity of the field (Figure 1). Faraday conceived all space to be filled with such magnetic force fields: 'I cannot refrain from again expressing my conviction of the truthfulness of the representation, which the idea of lines of force affords in regard to magnetic action' [11].

Scottish mathematician James Clerk Maxwell read Faraday's Experimental Researches and was struck by the lines of force concept. In 1855-56 Maxwell developed mathematical models to characterize magnetic field properties and better understand its physical nature [12]. He concluded that light and magnetism are different manifestations of the same substance, i.e., aether, and that light is an electromagnetic phenomenon that propagates through the field: 'I want to be understood literally . . . energy resides in the electromagnetic field, in the space surrounding the electrified and magnetic bodies, as well as in these bodies themselves' [13]. The field is the repository of energy. What Maxwell is saying can be illustrated in a simple example: why does a lightbulb filament glow? It is not due to electricity flowing through the filament per se but, rather, because as the current flows field energy is drawn into the filament from the encompassing medium. In 1856 William Thomson reported the rotation of the plane of polarization of light by magnetic fields and deduced that the field had a rotatory character and possessed angular momentum, i.e., force. Maxwell seized on this idea and came to regard the magnetic field as consisting of rotating vortices with electric currents representing a 'species of translation' which, like light, possessed linear attributes. The recognition that light, electricity and magnetism are different manifestations of a single phenomenon led Maxwell to deduce the existence of an aethereal medium that fills all space and permeates solid bodies [14].

To paraphrase late-19 $19^{\text {th }}$ century physicist Oliver Lodge, there cannot be waves unless they are waving in something. While most physicists accepted magnetic and electric phenomena as forces arising from aether, or that it was responsible for the propagation of light, there was no consensus as to its nature or how such translational events took place. Some considered aether to be a continuous, motionless, inertial medium that generated force when perturbed; others, like Maxwell, considered it to be composed of more than one constituent, a system of vortices and spinning particles; Thomson regarded it as a fluid, coining the term 'vortex sponge' to explain how polarized and non-polarized areas may coexist within an otherwise homogenous medium. But, as Thomson remarked near the end of his career, he knew little more about the relationship between magnetism, electricity, matter, and aether than when he had begun his studies fifty years earlier.

The 200-year saga of the luminiferous aether ended abruptly in 1887 with the infamous Michelson-Morley experiment [15]. The idea behind the experiment was that as the earth moved through the stationary aether (supposedly creating an 'aether wind' due to its subtle density) the speed of light should be different along the 
axis of earth's movement than in the perpendicular direction. Using a sensitive device with sufficient resolution to detect the calculated result, they found no difference. Regardless of which direction the light was directed it always traveled at the same speed indicating lack of relative motion between earth and aether.

Over the course of two centuries the aether concept morphed from Aristotle's insensible celestial substance, essential for the circular motion of the heavenly bodies, to an all-encompassing principle necessary to explain the action of gravitational forces and propagation of electromagnetic waves. The material form of aether had been sought out experimentally by scientists over the course of centuries without the least success. While the null result of the Michelson-Morley experiment was taken by many to refute the existence of aether, in reality it simply confirmed that the aether scientists had postulated did not exist. The luminiferous aether was a theoretical fiction that had engaged the best and brightest scientific minds to no avail. As early Greek philosopher Heraclitus wrote, nature loves to hide.

\section{Relativity \& Aether}

In 1905, Einstein published his piece On the Electrodynamics of Moving Bodies and in a subsequent lecture asserted that 'we regard the aether hypothesis as an obsolete point of view' [16]. In his hugely influential paper Einstein sought to address incompatibilities between classical Newtonian mechanics, Maxwell's demonstration of the wave-like basis and intertwined nature of light, electricity, and magnetism, and the null result of the Michelson-Morley experiment. Einstein, it appears, had been swayed by popular opinion.

Einstein's special theory of relativity examined relationships between Newtonian space and time as revealed from the perspective of light waves, which had been earlier calculated to travel at $186,000 \mathrm{~m} / \mathrm{s}$. He assumed that the laws of physics are the same for all reference points regardless of the state of motion and that the speed of light in a vacuum is always constant regardless of the motion of light or of the observer. By the very assumptions embedded in Einstein's revolutionary thesis it was destined to upend classical Newtonian mechanics and any established notion of absolute space, time and motion.

Einstein's paper, however, did not systematically address the aether issue and his conclusions concerning its existence apply only to the mathematical line of reasoning he developed. In retrospect, however, despite his denial of aether, the concepts Einstein advanced concerning the nature of so-called space-time support its existence.

Einstein inferred on the basis of his mathematical models that as an object approaches the speed of light, i.e., relativistic velocity, established relationships between space and time become altered giving rise to relativity phenomena such as time dilation, disruption of simultaneity, length contraction, interconversion of matter and energy, and more. According to the state of motion of an object, time flows differently, distance becomes relative, and mass contracts.

Depending on their respective states of motion, events may appear to occur simultaneously to one observer and temporally separate to another. Based on Einstein's thesis, Newton's absolute time and space are only locally valid. Moreover, time and space do not exist separately and distinctly but, instead, are interwoven into a continuum called space-time. Einstein doesn't comment on the nature of this continuum but we can safely assume it cannot exist in empty space.

In the simplest sense, what relativity seems to hint at is that conditions preceding space, time or motion are absolute while all derived effects are relative and pointof-view dependent. In his equations Einstein held the speed of light to be constant and thus by default it became the implicit frame of reference. No matter what slice of spacetime from which the absolute is observed it always appears absolute. Such preconditions are satisfied if one considers space to represent a boundless resonance field in which the whole is simultaneously present everywhere.

Beginning with the phenomenon of light Einstein sketched out implications regarding a wide range of physical phenomena, many of which have been experimentally validated over the course of the $20^{\text {th }}$ century. But a halfcentury earlier Maxwell had established that light is but one aspect of the electromagnetic spectrum and while it may have certain unique features, i.e., pointlike properties, that distinguish it from magnetism they are still intertwined manifestations of the same primary phenomenon. To explain the conjoined nature of such energetic phenomena experimental physicists from Newton through Maxwell were unanimous as to the necessity of an all-encompassing medium, aether, by which cause-and-effect is mediated. However, one may conceive light, electromagnetic phenomena require the aethereal medium. Action-at-a-distance is impossible.

Ten years later, apparently after he had time to think things through more carefully, Einstein did an abrupt about-face and changed his perspective on aether. This coincides with publication in 1915 on general relativity in which he attempted to incorporate gravity into his relativistic framework. In this work Einstein reinterprets Newton's universal law of gravitation and offers a new view of gravity as a geometric property of relativistic space-time. A consequence of this formulation is the curvature of the space-time continuum related to the presence of energy and matter. It is difficult to imagine 
how empty space can curve. General relativity would seem to mandate an aethereal medium.

In the process of redefining gravity Einstein reinvented aether: 'The special theory of relativity does not compel us to deny aether. We may assume the existence of an aether, only we must give up ascribing a specific state of motion to it .. .' [17]. This has far-reaching implications. Einstein strips away any and all mechanical attributes of aether: it has neither material nor temporal properties. It can only represent an indefinable inertial medium. A worse possible scenario for experimental science couldn't be imagined: aether is completely refractory to demonstration or quantification (as 350 years of scientific investigation attest). Einstein's aether begins to resemble that of Aristotle.

As an omnipresent inertial medium, aether is the ground for all observed physical spatial phenomena, affecting properties of all bodies at any given point. Einstein affirms Aristotle's doctrine of no action-at-a-distance: 'to deny aether is to ultimately assume that empty space has no physical qualities whatsoever. The fundamental facts of mechanics do not harmonize with this view . . ' [18]. In general relativity action can only take place within a continuous field and this continuum by default must represent aether.

In his insightful essay Aristotle's Aether and Contemporary Science (2004) philosopherChristopher DeCaen comments, 'aether's executioner turns out to be its saviour' [19]. By the same token Einstein became the unwitting executioner of contemporary atomistic philosophy. Atomism as a tenable doctrine is dead.

But Einstein had little to say about the positive attributes of aether. If it is utterly transparent, insensible, has no physical or temporal (motional) properties whatsoever and can, at best, be described as an inertial medium then how can it be said in a proper sense to even exist? As Charles Steinmetz and Nicola Tesla argued a century ago, aether can only be conceived as an all-pervasive resonance medium.

\section{Quantum Vacuum \& Aether}

After relativity the other dominant framework in modern physics is quantum theory. While Einstein's relativity applies to the macro-universe, quantum physics concerns phenomena at the atomic and sub-atomic scale-but only to the extent that parameters like wavelength, velocity and mass are detectable. Just as Einstein was forced to recognize an aetheric medium to explain gravitational and electromagnetic fields, so too has quantum physics been increasingly drawn toward the continuum point-of-view. The most compelling evidence comes from a branch of physics known as quantum electrodynamics (QED).
As Newtonian physics was torn between particle and wave models of light, the wave-particle duality continues to haunt quantum physics. Objects at the quantum level have dual attributes: they possess wavelength, frequency and disperse through space like waves but are discrete and localized like particles. Quantum events are probabilistic and what happens in any given experimental circumstance cannot be predicted with certainty, a phenomenon known as the uncertainty principle. This inherent uncertainty reflects not only limitations of scientific knowledge but, equally, imputes an underlying indeterminacy of waveparticle phenomena themselves.

Equally problematic as wave-particle duality is the nature of the medium in which such phenomena reside. The term vacuum state refers to the lowest possible energy content of the quantum field, one in which no particles are supposed to be present. Werner Heisenberg, who advanced the uncertainty principle, argued in the 1920s that the vacuum must contain residual irremovable energy, which came to be called zero-point energy.

Given that random signals are generated from within the vacuum space by transitory electromagnetic phenomena, the so-called vacuum state cannot really be empty. Particles, or energy packets with mass-like attributes, i.e., 'virtual particles,' emanate from this fluctuating medium [20]. Even though there is supposedly nothing in the field there is always the possibility that something will come out of that nothingness. This suggests the presence of something, a state of empty-fullness that Aristotle called the plenum.

The most commonly cited example in support of the fullness of the quantum vacuum and zero-energy state is the Casimir effect $[21,22]$. Two uncharged conductive plates are placed in a vacuum in nanometer proximity to each other. If no field were present between the plates, then no force should be measured. But numerous experiments show a quantifiable attractive or repulsive force between the plates.

While manifesting at the sub-atomic level, the Casimir effect is not dissimilar (or unrelated) to Faraday's original experiments: the presence of conducting materials, i.e., dielectrics, alters dynamics within the surrounding field. The presence of attraction and repulsion of the plates imputes the presence of a magnetic force. While attempts have been made to explain the effect in terms of particle interactions, zero-point energy and field theory is more applicable. How to explain the Casimir effect without invoking aether and field dynamics? Equally compelling evidence emerges from other well-known QED phenomena.

The term 'entanglement' was coined by Erwin Schrödinger in 1935 to refer to particles that interact, move off in separate directions, and yet still influence each other's behaviours' [23]. The possibility of such paradoxical 
effects (paradoxical in the sense of classical Newtonian mechanics) had been raised in a paper by Einstein, Podolsky and Rosen earlier that year [24]. Schrödinger called entanglement 'the characteristic trait of quantum mechanics'. Unsettled by the notion Einstein called it 'spooky action at a distance'. Quantum entanglement was experimentally validated in 1949 [25].

Quantum entanglement describes interactional dynamics that occur when groups of atomic or sub-atomic 'particles' are generated or share spatial proximity such that the quantum state of each particle in the cluster cannot be separated from the state of all the others. Measurement of physical properties of such particles, i.e., spin, momentum, and position often closely correlate. In entangled systems if one particle possesses a clockwise spun a second particle along the same axis will spin in the counterclockwise direction with both possessing the same angular momentum, giving an inverse or mirror-image energy symmetry so that the net difference between the two vectors is always zero. But zero does not connote nothing.

It has been observed that once a measurement of polarization or spin is made on one of the entangled particles the wave function of the entire system collapses before either of the so-called particles can directly interact. Such phenomena have been replicated even when particles are widely separated and suggest that communication between entangled particles takes place at speeds greater than that of light (which is to say instantaneously) [26, 27]. By necessity such results impute collective field mediated behaviours and the presence of an organized resonance structure.

These behaviours were anticipated in a 1951 article in Nature by physicist Paul Dirac, founder of QED [28, 29]. Dirac argued that aether had been prematurely rejected. Recognizing the impossibility of an absolute void, he wrote that physicists must make 'profound alterations' in their theoretical conception of the vacuum. Based on emerging evidence in electrodynamics, he claimed, 'we are forced to have an aether'.

According to relativity theory the aether medium must be motionless and homogenous, i.e., having the same properties in all directions, but in adapting the aether to QED, Dirac's aether, as opposed to that of Einstein, possessed superluminal, i.e., greater than the speed of light, velocities and engendered absolute simultaneity of cause and effect. Such instantaneous cause and effect can only be explained on the basis of a resonance medium in which velocity becomes irrelevant.

Dirac advanced a radical departure from the pointlike atomic model to a non-local 'extended' particle, thus removing the last vestige of atomism that had characterized western science since its inception in the $17^{\text {th }}$ century [30]. So-called particles propagate instantaneously on the basis of internally generated superluminal dynamics and represent a point charge embedded in its own radiating electromagnetic field. The atomistic notion of the electron as particle was but science fiction. In recent years an increasing number of scientists and philosophers are coming to the same conclusion as Dirac seventy years ago: science can no longer progress without the aether concept [31].

\section{Wheeler's Aether}

In the last decade a blistering assault on the bulwark of modern physics, ranging from Newtonian mechanics through relativity and QED, has come seemingly out of nowhere from an unexpected source, philosopher and physiologoi Ken Wheeler, whose magnum opus Uncovering the Missing Secrets of Magnetism (2014) obliges a complete revision of atomistic scientific dogma [32].

Wheeler advances the most comprehensive and integrated dynamic framework in western science to date detailing the intertwined nature of aether, fields, and energy dynamics. His aether-centric explanation of fields redefines the nature of space, time and motion and, as opposed to the speculative theories of modern atomistic science, constitutes a rigorous cosmology based on the necessity of aether and the continuum. Given that scientific knowledge advances largely on the basis of reframing of outdated concepts and refutation of existing theory, his critique of relativity and quantum mechanics is highly relevant. The scope and power of Wheeler's framework suggests that he will have the last word on the aether question for the foreseeable future.

If you want to rattle a physicist, says Wheeler, ask him/her to define a field. The field, as conceived by modern science, is an abstract particle-based nexus derived from erroneous notions of space and time, i.e., space-time, which, because it does not include an aethereal medium, cannot explain how charge, gravitational and electromagnetic forces, or distant actions are mediated.

The field, a dynamic continuum, originates in the aether and consists of aether. By its nature the field entails spatial extension but the relationship is hierarchical: the field does not exist in space but rather space exists within the field. Neither space nor time, as postulated by Newton, are absolute but exist only in relation to mass, magnitude, distance and motion. The field, on the other hand, is a mass-free non-physical medium. Nor does field represent force. Instead, force derives from tension or torque at the aether boundary. Aether, non-spatial, non-temporal, frictionless, invisible, and undetectable, exists in a state of rest (inertia) unless disturbed at which time it discharges as force or motion. 
The field manifests in the modalities of dielectricity, electromagnetism, mass and gravity, all nuanced variations of aether characterized by their attributes, i.e., wave-like behaviors, spatial geometry, or force properties. Fields assert their effects through motion and pressure. Nature, says Wheeler, doesn't work in straight lines but in spirals and manifests in dynamic oppositional pairs such as centrifugal-centripetal, clockwise-counter clockwise, circular-radial, and spatial-counter-spatial. Motion, a pressure discharge, takes origin in the dimensionless inertia of the aether field. Moreover, all force and motion terminate not into space but rather back into the aether. All states of equilibrium imply an aetheric medium. Such phenomena raise doubts regarding Newton's supposed laws of motion.

The easiest way to understand aether dynamics is to consider the spatial and counterspatial interactions of its two primary modalities, dielectricity and magnetism, which Wheeler calls the conjugate field, implying an entangled and inseparable coexistence. Given that everything originates in aether the very presence of space implicates a polarized field comprising two opposing forces and motions: the dielectric, with counterspatial and contractive tendencies, and the magnetic, with spatial and expansile attributes.

With dielectricity, force lines are directed inwardly along the counterspatal axis toward the aether medium. Dielectricity represents the aether boundary, the inertial plane which produces stress and torque thus generating aether-mediated effects. In electrical parlance this involves the phenomena of conduction and capacitance. As Eric Dollard notes, the concept of capacitance as an electrical current that charges a conductor with a quantity of electricity is inadequate to explain capacitance [33]. In capacitance the dielectric field draws conductors into tighter spatial apposition thus increasing counterspace and actuating aether flux. How else to explain the paradoxical capacitance phenomenon that the smaller the space bounded by the conducting structure the more energy that can be 'stored,' i.e., drawn from the aether medium?

Counterspace represents the so-called vacuum or zeropoint fulcrum of QED through which all field phenomena manifest. Dielectricity thus precedes all the other modalities in the hierarchical manifestation of aether effects not to mention force unification. Dielectricity terminates in the formation of objects possessing mass and magnitude of which gravity is a by-product. Through the centripetal and radial flow of dielectricity in conductor substances the currents ultimately reflect upon themselves to form the outwardly-directed 3-D phenomenon of magnetism.

As Maxwell showed, magnetism does not exist as a standalone entity but is part of the intertwined spectrum of electromagnetic phenomena and can only be induced via field interactions. Magnetism originates in the dielectric and, like matter, represents one of its discharge modalities. Magnetism is the only aether modality to possess spatial dimensions and thus space itself can only be an attribute of magnetism. Magnetism represents the polarization of dielectricity when in contact with matter or induced by electricity. To say magnetism is spatial is to say that as the polarized field expands out of counterspace it creates open (but not empty) space.

Dielectricity and magnetism, as mechanical forces, are complementary and inverse in their field architecture: One is counter-spatial, radial, centripetal and contractionary; the other is spatial, circular, expansile and possesses both centrifugal and centripetal components. The expansile radiative movement of magnetism away from the dielectric begins as a circular centrifugal motion that assumes spherical dimensions and returns into the aether field via a centripetal movement. These two coprinciples of the conjugate field form the yin and yang of what physicists call space-time, representing universal principles of force and motion based on aether inertia and field induction.

As Faraday observed in his experiments, simply waving a magnet across a conductor induces current flow. And as Maxwell later commented, electricity represents a 'species of translation'. Electricity, a hybrid aether modality, arises at the interface between the dielectric and magnetic fields. As the sweeping magnet passes the dielectric conductor a torque is generated from the interacting magnetic and dielectric field oscillations along both sides of the of the inertial plane which disturbs the dielectric equilibrium at the atomic and interatomic levels causing electrification along a plane perpendicular to the two.

Electrification is the result of membrane torque and dynamic radial polarization of the aether. Given that electrification of ferrous objects induces a magnetic field it is evident that electricity must terminate in magnetism by virtue of its interaction with a dielectrically capacitant object. In the second part of this paper, we will show that these same effects are at play in living bodies.

In electrification the discharge plane occurs at the atomic level and involves the electron which Wheeler, as Dirac before him, insists is pure atomistic fiction. Rather than representing a charged particle, the so-called electron is the fleeting effect of the interaction between the dielectric and magnetic fields at the atomic level: the atomic nucleus is composed of both dielectric and magnetic fields and the electron shell, with its hypothetical circular spatial orbitals, simply represents electricity in motion. And as Wheeler shows, the dynamic architecture of the macromagnetic field is also a result of conjugate interactions 
between the magnetic and dielectric forces mediated by electricity (See Figure 1).

The magnetic field is doughnut-shaped (toroidal) with complete polarization, i.e., top-to-bottom and insideto-outside inverse symmetry, of its topographic relations resulting in centrifugal (divergent) and centripetal (convergent) motions of magnetic force lines. The very same point in the field which is divergent in one region becomes convergent at a point 180 degrees on the opposite side. A counter-clockwise directional spin on the inside of the torus translates into a clockwise spin on its outer surface. Clockwise and counterclockwise, centrifugal and centripetal, are variations of the same primary motion and is point-of-view dependent. Such dynamic field architecture explains quantum entanglement or the collapse of the entire field function when a measurement to determine the position of a so-called particle is made.

As the magnetic force lines cannot make contact and merge into one another they seek equilibrium by spherically corkscrewing towards the opposite pole and terminating back into the aether field. Such force line behaviours impute the presence of a counterforce that resists convergence of the expansile 3-D magnetic field lines within the spatial domain. The same force that causes adjacent lines of similar spin to repel at the periphery of the doughnut also accounts for the repulsion effect when two north poles or south poles of the magnet come into proximity. This counterforce, representing the tendency toward outward movement and spatial expansion, is entwined into the 3-D geometry of the magnetic field and opposes the counter-spatial tendencies of dielectricity and gravity. As the dielectric comes out of the aether, and the magnetic out of the dielectric, the magnetic terminates back into the aether. Magnetism (like any force or motion) seeks geometric and spatial equilibrium in the aether.

At the equator of the torus, between the polar divergent and convergent magnetic flux lines, lies the dielectric inertial plane which functionally opposes the spatial magnetic force. The magnetic and dielectric field geometries interact in an inverse manner to generate electrical currents. As the magnetic field expands it induces an electrical discharge which courses into the counterspatial dielectric causing its rebound contraction and generating torque at the inertial plane that results in a transient hour-glass deformity of the radiating magnetic field.

The generation of an external magnetic field, as in a permanent magnet or transiently during magnetic resonance imaging (MRI), deserves special mention. During electrification as current streams into the nucleus it produces dielectric saturation with resultant coherent in-phase precession of countless protons at the inertial plane producing enormous torque. As the dielectric and magnetic fields are incommensurate and mutually repellant, the intra-nuclear magnetic field is literally extruded into the inter-atomic space assuming 3-D macro-magnetic predominance surrounding a magnetized object.

Dielectrically induced coherency, in which nuclei align and precess uniformly to generate compounded angular momentum, is the driving force behind attraction and repulsion phenomena of magnets. As Wheeler points out, however, magnetic attraction and repulsion are misnomers: when two magnets are said to attract one another the dielectric inertial plane spontaneously relocates itself to the interface between the two magnets and space is thereby voided. When two north or south poles repel one another, what he calls counter-voidance, the expansionary forces of the two magnets come into proximity and resist further spatial voidance. Attraction and repulsion, like the terms' sunrise and sunset, are based on erroneous conceptions of natural phenomena.

The unfolding dynamics of aether and the conjugate dielectric/electromagnetic field, as described accurately and comprehensively for the first time ever by Wheeler, point an incriminating finger at 350 years of speculative experimental science. Newton's first law of motion, as Wheeler points out, is 'utterly defunct' and fails to explain the origin and nature of force and motion, the distinction between field and force, or accurately describe inertia as a primary attribute of aether.

By the early $19^{\text {th }}$ century Newton's corpuscular theory of light had been discredited in favor of wave theory and yet for over two centuries' scientists have persisted in erroneously ascribing electromagnetic phenomena to hypothetical particles like electrons and photons, even engaging in a prolonged and futile search for the so-called 'god particle'. As Wheeler points out, light and electricity are utterly mass-free. All particle-based conceptions of such dynamic phenomena are impossible and solely a product of the defunct atomistic perspective.

Central to the shortcomings of atomistic science is its failure to correctly explain the nature of the field, which Wheeler calls a 'titanic omission'. Everything is field and everything comes out of field, which is only aether. Einstein and his acolytes argued that fields and space were equivalent but, as Wheeler shows us, to use space to define a field is a monumental category error unparalleled in western intellectual history. Quantum and relativity, he claims, is a 'quack religion' based on the absurd premise that the universe is a sea of massless particles moving through empty space. Equally absurd, he argues, is Einstein's notion of curved space: 'only a warped mind could conceive of warped space'. As we have seen, the only viable explanatory framework entails recognition of a universal continuum constituted by the aether field. All else fails. 


\section{Aether \& the Continuum}

We have examined the nearly 2400 years history of the aether concept, from Aristotle through Wheeler, and seen its evolution from an insensible, diaphanous substance occupying the celestial realm, responsible for the circular motion of heavenly bodies, to an all-encompassing medium pervading the entirety of the cosmos, from the stars down into the sub-atomic domain.

Despite the inability of 350 years of scientific experimentation todemonstrateaether its effects continue to beleaguer and torment contemporary scientists. While aether cannot be sensed, and is undetectable by even the most delicate of instruments, it remains conceptually indispensable to understand the nature of a multitude of observed celestial and terrestrial phenomena. In this sense, despite his incomplete description of its effects and underestimation of its universal extension, science has come full-circle back to Aristotle's aether and the necessity of the continuum. Atomism is indisputably dead. In the second part of this paper we examine the spectrum of aether-induced effects in living bodies.

Figure 1: Faraday's lines of force.

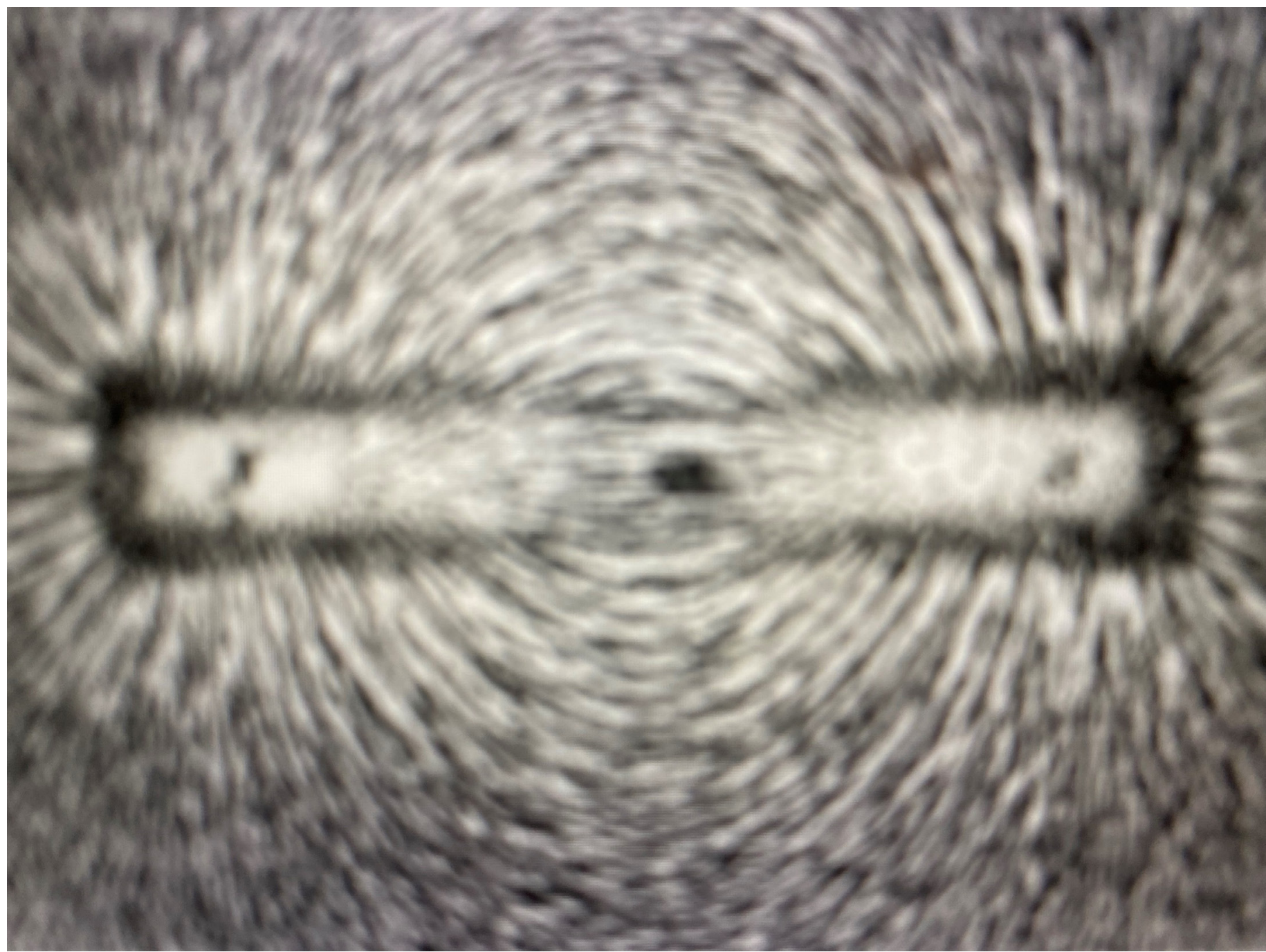


Figure 2: Wheeler's integrated model of the conjoined magneto-dielectric field. [White $=$ Aether; Blue $=$ Magnetism; Red = Dielectric; Yellow = Electricity]

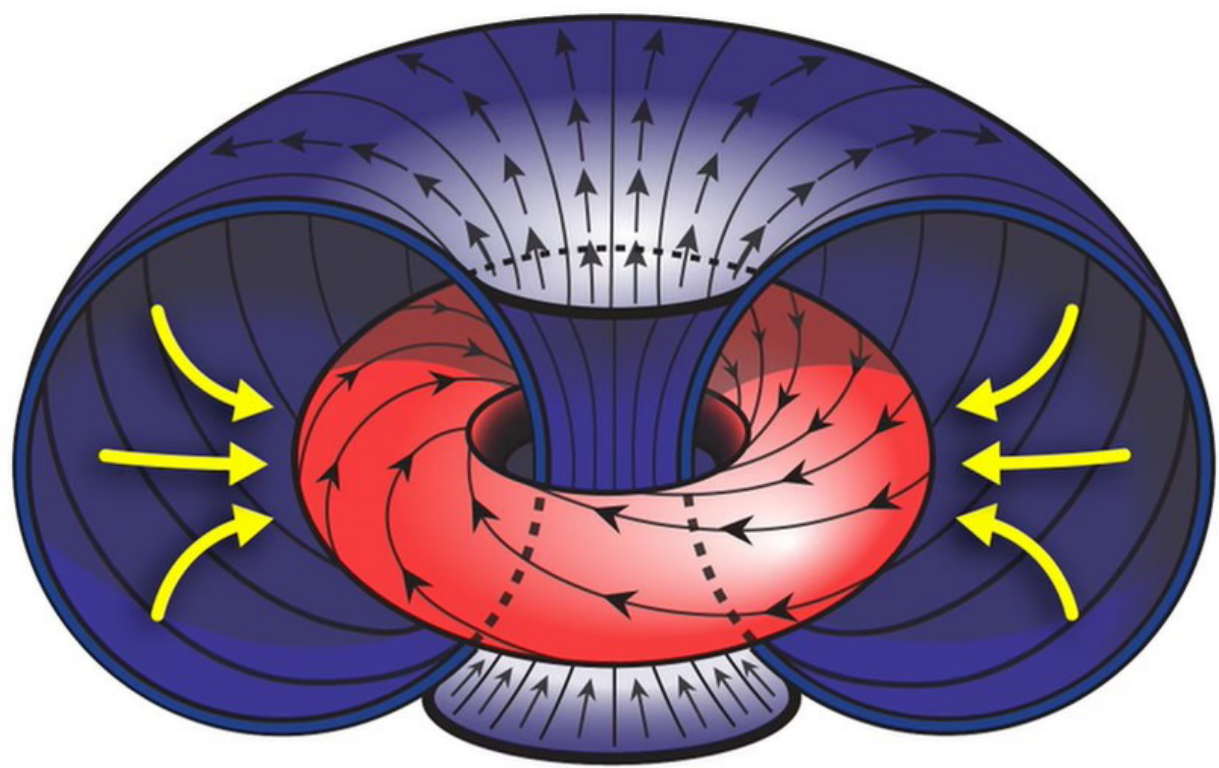

\section{References}

1. On the Heavens. In: The Complete Works of Aristotle. Revised Oxford Translation (Vol. 1) ed. Jonathon Barnes. Princeton University Press 1984

2. The Cambridge Companion to Aristotle. ed. Jonathon Barnes Cambridge Univ. Press 1995

3. Isaac Newton: Letters to Bentley, $1692-93$ cited in: Action at a Distance in Quantum Mechanics. The Stanford Encyclopedia of Philosophy: Berkovitz J., 2008

4. A History of the Theories of Aether and Electricity-From the Age of Descartes to the Close of the Nineteenth Century Edmund T. Whittaker publ. Forgotten Books 2018; pp. 1-28

5. Newton's Revival of the Aether Hypothesis and the Explanation of Gravitational Attraction. Hawes JL
Royal Soc J Hist Sci 23(2): Dec 1968

6. Newton's Views on Space, Time, and Motion. In: The Stanford Encyclopedia of Philosophy Rynasiewicz, R. 2011

7. Robert Boyle's Landmark Book of 1660 with the first experiments on Rarified Air. West JB J Appl Physiol. Jan 2005; 98(1): 31-39

8. A History of the Theories of Aether and Electricity-From the Age of Descartes to the Close of the Nineteenth Century. Edmund T. Whittaker publ. Forgotten Books 2018; 1-28

9. ibid., 105-110

10. ibid., 188-221

11. ibid., 191

12. A Treatise on Electricity and Magnetism. Chapter 
XX. James Clerk Maxwell

13. A History of the Theories of Aether and Electricity-From the Age of Descartes to the Close of the Nineteenth Century. Edmund T. Whittaker publ. Forgotten Books 2018; pp. 268-309

14. ibid., 310-336

15. The Michelson-Morley Experiment. Michael Fowler. http://galileoandeinstein.physics.virginia.edu/ lectures/michelson.html

16. The Development of Our Conception of the Nature and Constitution of Radiation. Albert Einstein. Presented at the session of the Division of Physics of the 81st Meeting of German Scientists and Physicians in Salzburg on September 21, 1909.

17. Albert Einstein. Sidelights on Relativity. Publ. Dover, New York 1983, 13-15

18. ibid., 16-17

19. Aristotle's Aether and Contemporary Science. Christopher A. DeCaen. The Thomist, 2004; 68(3): 375-429

20. Are virtual particles really constantly popping in and out of existence? Or are they merely a mathematical bookkeeping device for quantum mechanics? Scientific American. October 9, 2006

21. On the Attraction Between Two Perfectly Conducting Plates. Casimir HBG. Indag Math. 1948 10: pp 261-263 (also in Front Phys 1987 65: 342-44)

22. Science and Technology of the Casimir Effect. Stange A, Campbell D, Bishop D Physics Today 2021: 74(1): 42

23. Discussion of probability relations between separated systems. Schrödinger E Math Proc Cambridge Philo Soc. 31 (4): 555-563.
24. Can Quantum-Mechanical Description of Physical Reality Be Considered Complete? Einstein A, Podolsky B, Rosen N Phys Rev 47(10): 777-780

25. The Angular Correlation of Scattered Annihilation Radiation. Wu CS, Shakov I Phys Rev. 1950; 77(1): 136

26. Bounding the Speed of 'Spooky Action at a Distance.' Yin J, Cao Y, Yong H-L, Ren J-G et al. Phys Rev Lett. 2013; 110(26): 260407

27. Cosmic Test Bolsters Einstein's 'Spooky Action at a Distance.' Elizabeth Gibney. Nature. February 3, 2017

28. Is there an Aether? Dirac PAM Nature 1951; 168 : 906-7

29. Quantum Mechanics and the Aether. Dirac PAM. Sci Monthly. 1954; 78(3): 142-46

30. Dirac's Aether in Relativistic Quantum Mechanics. Petroni NC, Vigier J-P In: Quantum, Space and Time: The Quest Continues Eds. A. O. Barut, A Van Der Merwe, J-P Vigier Cambridge Univ Press, 1984

31. Revisiting the Aether in Science. Rubik B, Jabs $\mathrm{H}$ Cosmos and History: The Journal of Natural and Social Philosophy 2018; 14(2): 239-255

32. Uncovering the Missing Secrets of Magnetism.Ken Wheeler. https://ia802502.us.archive.org/31/items/magnetism1small/magnetism1small.pdf

33. Introduction to Dielectricity \& Capacitance. Eric P. Dollard. http://ericdollard.com 